\title{
Cardiac magnetic resonance findings predicting mortality in patients with pulmonary arterial hypertension: a systematic review and meta-analysis
}

\author{
Vivan J. M. Baggen ${ }^{1,2} \cdot$ Tim Leiner $^{3} \cdot$ Marco C. Post $^{1} \cdot$ Arie P. van Dijk $^{1}$. \\ Jolien W. Roos-Hesselink ${ }^{2}$ - Eric Boersma ${ }^{2,4}$. Jesse Habets ${ }^{3}$. Gertjan Tj. Sieswerda ${ }^{1}$
}

Received: 17 August 2015 / Revised: 4 January 2016 / Accepted: 13 January 2016 / Published online: 4 February 2016

(C) The Author(s) 2016. This article is published with open access at Springerlink.com

\begin{abstract}
Objectives To provide a comprehensive overview of all reported cardiac magnetic resonance (CMR) findings that predict clinical deterioration in pulmonary arterial hypertension (PAH).

Methods MEDLINE and EMBASE electronic databases were systematically searched for longitudinal studies published by April 2015 that reported associations between CMR findings and adverse clinical outcome in PAH. Studies were appraised using previously developed criteria for prognostic studies. Meta-analysis using random effect models was performed for CMR findings investigated by three or more studies.

Results Eight papers (539 patients) investigating 21 different CMR findings were included. Meta-analysis showed that right ventricular (RV) ejection fraction was the strongest predictor of mortality in PAH (pooled HR 1.23 [95 \% CI 1.07-1.41],
\end{abstract}

Electronic supplementary material The online version of this article (doi:10.1007/s00330-016-4217-6) contains supplementary material, which is available to authorized users.

Gertjan Tj. Sieswerda

G.T.Sieswerda@umcutrecht.nl

1 Department of Cardiology, AHMaZON Centre for Adult Congenital Heart Disease, University Medical Centre Utrecht, Radboud University Medical Centre Nijmegen and St. Antonius Hospital Nieuwegein, PO Box 85500, 3508 GA Utrecht, The Netherlands

2 Department of Cardiology, Erasmus Medical Centre, Rotterdam, The Netherlands

3 Department of Radiology, University Medical Centre Utrecht, Utrecht, The Netherlands

4 Department of Clinical Epidemiology, Erasmus Medical Centre, Rotterdam, The Netherlands $\mathrm{p}=0.003)$ per $5 \%$ decrease. In addition, RV end-diastolic volume index (pooled HR 1.06 [95\% CI 1.00-1.12], $\mathrm{p}=0.049$ ), RV end-systolic volume index (pooled HR 1.05 [95 \% CI 1.01$1.09], \mathrm{p}=0.013$ ) and left ventricular end-diastolic volume index (pooled HR 1.16 [95\% CI 1.00-1.34], $\mathrm{p}=0.045$ ) were of prognostic importance. RV and LV mass did not provide prognostic information ( $\mathrm{p}=0.852$ and $\mathrm{p}=0.983$, respectively).

Conclusion This meta-analysis substantiates the clinical yield of specific CMR findings in the prognostication of PAH patients. Decreased RV ejection is the strongest and most well established predictor of mortality.

Key Points

- Cardiac magnetic resonance imaging is useful for prognostication in pulmonary arterial hypertension.

- Right ventricular ejection fraction is the strongest predictor of mortality.

- Serial CMR evaluation seems to be of additional prognostic importance.

- Accurate prognostication can aid in adequate and timely intensification of PAH-specific therapy.

Keywords Pulmonary arterial hypertension · Prognosis . Magnetic resonance imaging $\cdot$ Meta-analysis $\cdot$ Mortality
Abbreviations
CMR Cardiac magnetic resonance imaging
HR Hazard ratio
LV Left ventricle/left ventricular
PA Pulmonary artery
PAH Pulmonary arterial hypertension
PH Pulmonary hypertension
RV Right ventricle/right ventricular 


\section{Introduction}

Pulmonary arterial hypertension (PAH) is defined as a mean pulmonary arterial pressure $\geq 25 \mathrm{mmHg}$ in the presence of a pulmonary capillary wedge pressure $\leq 15 \mathrm{mmHg}$ as assessed by right heart catheterization, and is characterized by progressive remodelling of the distal pulmonary arteries [1]. PAH includes apparent heterogeneous conditions (idiopathic, heritable, induced by drugs or toxins, associated with connective tissue diseases, HIV infection, portal hypertension and congenital heart disease), but is characterized by similar clinical, haemodynamic and pathological pictures [1,2]. Left untreated, the resultant increase in pulmonary vascular resistance leads to progressive deterioration of right ventricular (RV) function and eventually death in $45 \%$ of incident cases within 3 years $[2,3]$. Although recent advances in therapeutic modalities have significantly improved the outcomes of this devastating disease, the course of the disease widely varies between individuals [4-6]. In order to guide optimal clinical management of patients with $\mathrm{PAH}$, accurate prognostication and monitoring of disease progression is therefore of great importance [5].

Previously reported predictors of mortality include aetiology of PAH, sex and several functional, biochemical and haemodynamic variables [3, 7-10]. Although it is inferred that cardiac magnetic resonance imaging (CMR) could be of important additional value, evidence for the prognostic merit of specific imaging findings is still far from robust. This study therefore aims to provide a comprehensive overview of commonly investigated CMR findings that are predictive of adverse clinical outcome in PAH.

\section{Methods}

This systematic review was conducted in accordance with the PRISMA statement [11]. A pre-defined review protocol as adopted by this study can be accessed through PROSPERO (registration number: CRD42014009231).

\section{Literature search strategy}

CMR studies as described in this review were identified through a general search syntax that was designed to aggregate all studies concerning the prognostic value of noninvasive imaging in PAH. A comprehensive systematic search was performed on 29 April 2015 in MEDLINE (via PubMed interface) and EMBASE electronic databases using combinations of all synonyms for: population (PAH), non-invasive imaging and relevant clinical outcomes (components of the Dana Point Time To Clinical Worsening composite endpoint) $[2,12]$. A validated prognostic search filter with the highest sensitivity $(98 \%)$ was added to the search syntax $[13,14]$. No language or publication period restrictions were applied. The full original search syntax is supplied in Supplemental File 1.

\section{Selection of papers}

A flow diagram of the selection process is shown in Fig. 1 [11]. After deduplication, one author (VB) performed screening and selection of articles based on title and abstract, using the following exclusion criteria: cross-sectional study design, study population without $\mathrm{PH}$ (e.g. acute pulmonary embolism, exercise-induced $\mathrm{PH}$ ), $>30 \%$ study population with other than WHO group I PAH, inclusion of children $<12$ years of age, CMR findings not investigated as a potential predictor for clinical outcome, lack of Cox regression analysis. Full-text screening was performed by two authors $(\mathrm{VB}, \mathrm{JH})$; exclusion criteria are described in Fig. 1. All references of the excluded reviews and included articles were cross-checked to identify possible relevant articles missed in the original search syntax.

\section{Assessment of methodological quality}

Study quality was critically appraised using previously developed criteria for prognostic studies [15]. We assessed study design, missing data and loss to follow-up (selection bias), adequate description and measurement of imaging features and outcome (information bias), reported effect size, treatment of continuous risk predictors and multivariable adjustment for possible confounders.

\section{Data extraction and analysis}

Study characteristics and hazard ratios (HRs) for all investigated CMR findings with accompanying $95 \%$ confidence intervals (CIs) were extracted using a standardized form. Meta-analysis was performed for all CMR findings investigated by three of more studies, using random effect models. HRs for specific findings were recalculated to one uniform clinically applicable number of units change. Heterogeneity was assessed using Cochran's Q test and the $\mathrm{I}^{2}$ statistic [16]. CMR findings investigated as dichotomous variables were additionally presented in the corresponding forest plots. To determine the effect of individual study data, sensitivity analyses were performed by recalculating pooled HRs after excluding the results of one study. If study data were used in multiple papers and the same CMR findings were evaluated, only the study with the largest sample size was selected, thus excluding the risk of using duplicate data in our meta-analysis. The risk of publication bias was assessed using visual inspection of funnel plots and Egger's test [17]. 
Fig. 1 PRISMA (Preferred Reporting Items for Systematic Reviews and Meta-Analyses) 2009 flow diagram. * One study investigated both echocardiographic and cardiac magnetic resonance imaging (CMR) findings. $P A H$ pulmonary arterial hypertension
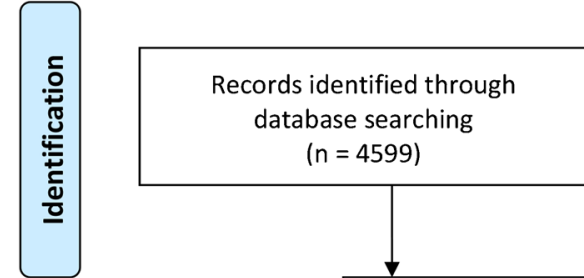

Additional records identified through other sources $(n=4599)$ $(n=1)$

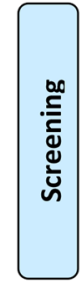

Records after duplicates removed $(n=2733)$
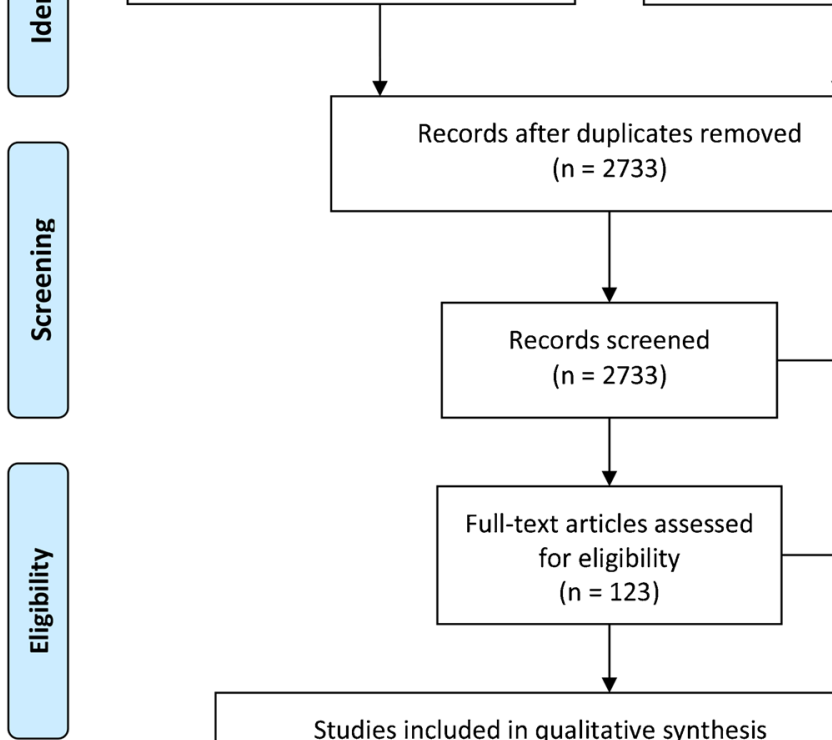

Studies included in qualitative synthesis $(n=44)$ : CMR findings $(n=8$, this review), echocardiographic findings $(n=37)^{*}$

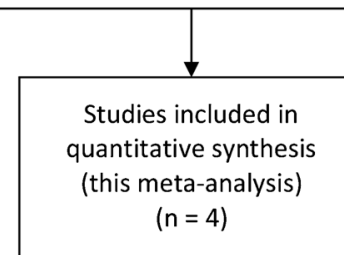

Records excluded $(n=2610)$ ( $n=79)$ : No non-invasive cardiac imaging parameters related to clinical outcome $(n=33)$, $>30 \%$ other than WHO group I PAH included $(n=26)$, no Cox regression analysis performed $(n=6)$, children

$(n=5)$, outcome after surgery/thrombectomy $(n=4)$, cross-sectional study design $(n=4)$, identical study cohort $(n=1)$

\section{Results}

\section{Search results}

Through a systematic literature search in MEDLINE and EMBASE and extensive reference cross-checking, 2,733 potentially relevant records were retrieved, of which 2,610 were excluded based on title and abstract. After full-text review of the remaining 123 articles, 44 papers were finally selected, of which eight studies in 539 patients described CMR findings (Fig. 1) [18-25].

Study and patient characteristics of the included studies are presented in Table 1. The studies were published between 2007 and 2014; study size ranged from 37 to 110 patients, mean age ranged from 39 to 62 years, and 60 $79 \%$ of the population was female. The majority of patients were diagnosed with idiopathic PAH (41-100\%), two studies included a subset of patients with congenital heart disease (9\% and $24 \%$, respectively) [23, 25] and one study included patients with PH group III, IV or V
(24\% of patients) [21]. Two studies did not report information on the use of PAH-specific mediation [24, 25]; in all other studies $>60 \%$ of patients were on PAH-specific therapy at baseline.

The majority of studies used death (or transplant) as the primary outcome; two studies used a composite outcome, additionally including hospitalization for heart failure [21, 23]. Mean follow-up duration varied between 10 and 45 months, and the primary outcome event occurred in 4-25 patients (10 $33 \%$ of the study population).

\section{Methodological aspects}

The individual bias assessment per study is detailed in Table 2 . Five out of eight studies had a retrospective study design. Information on missing values and loss to follow-up was not reported in two studies. It is therefore important to recognize the possible impact of selection bias.

Right heart catheterization was used for the diagnosis of PAH in $100 \%$ of the included patients. The majority 


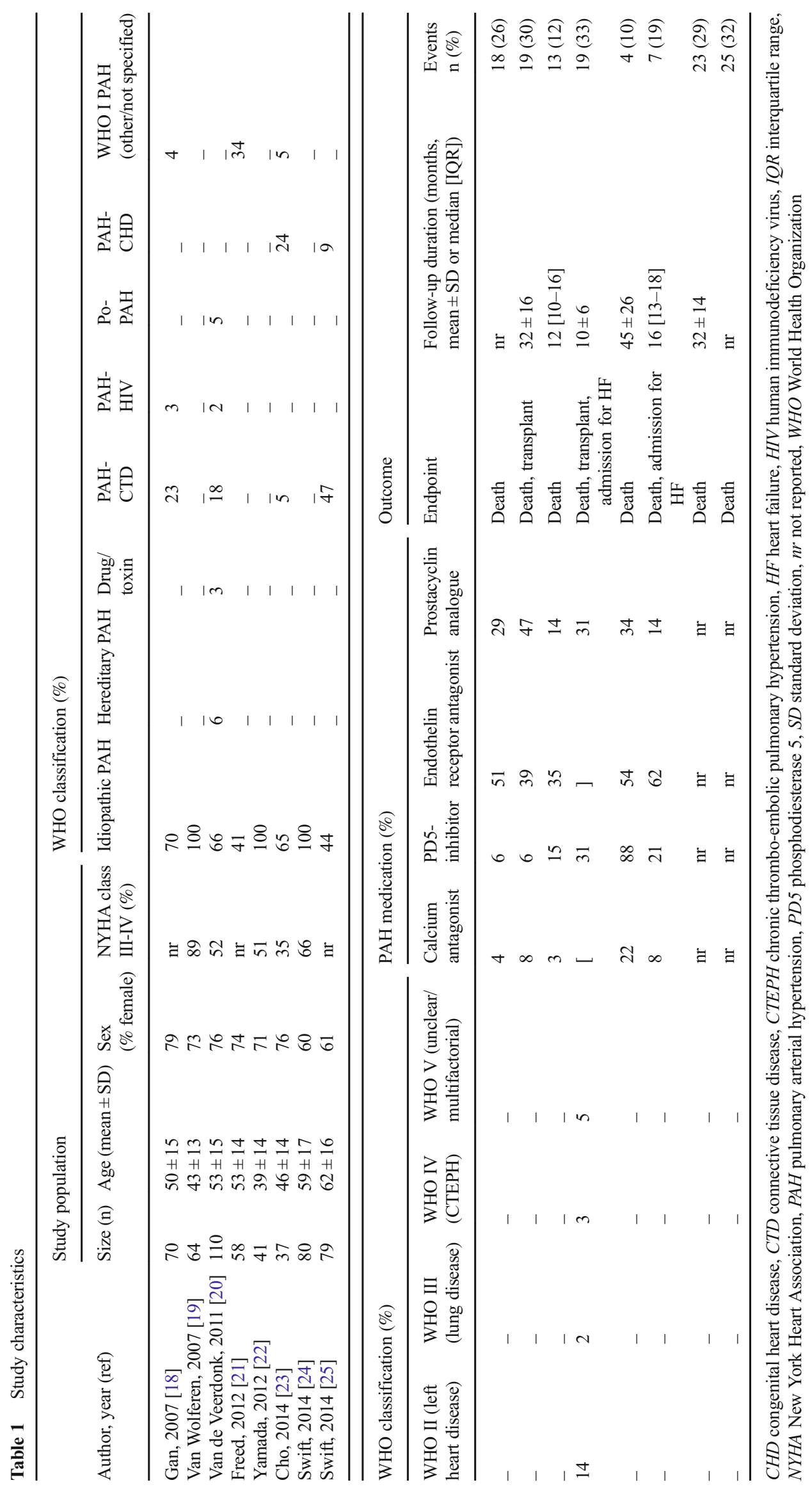




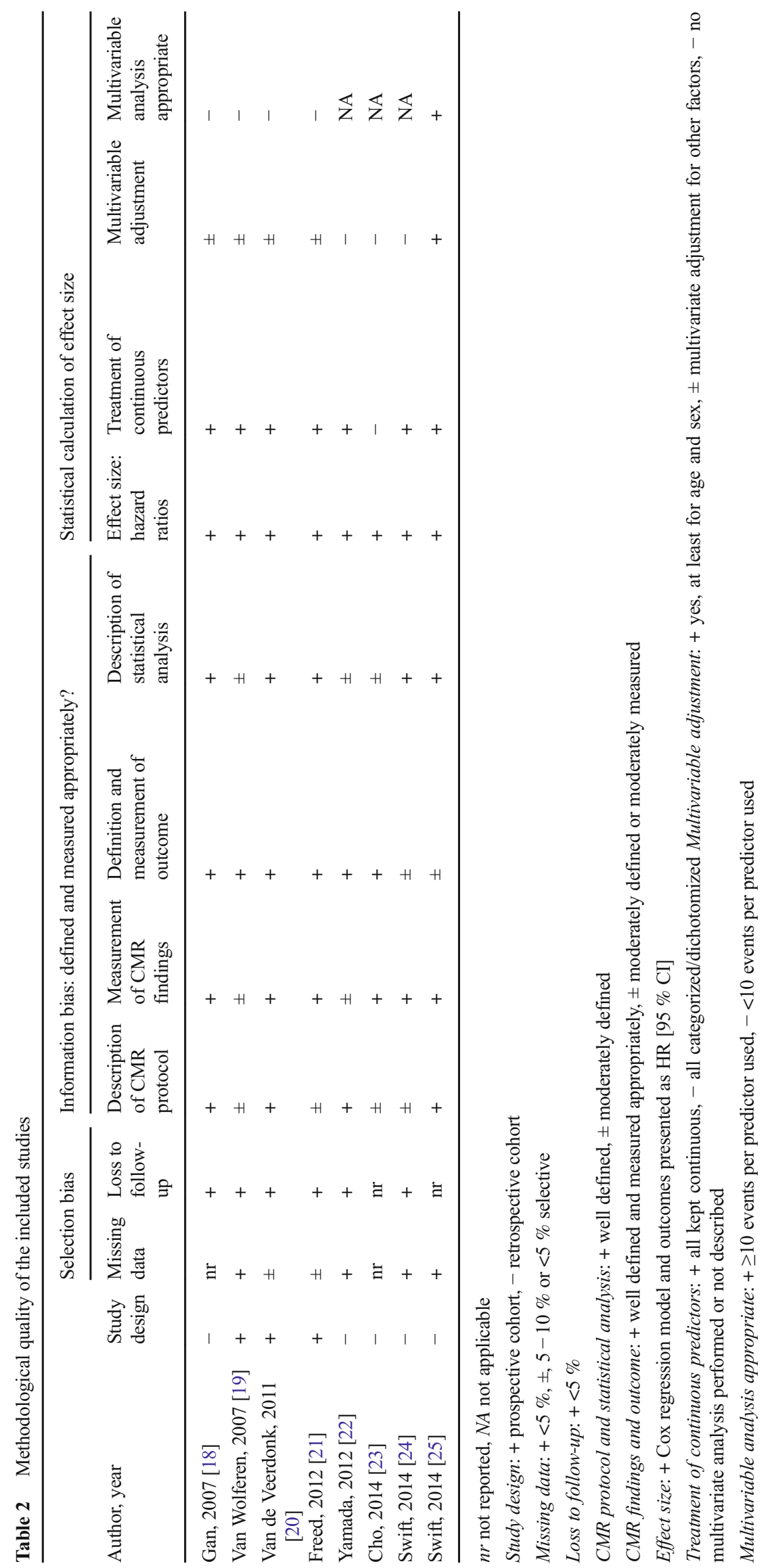


Table 3 Overview of investigated cardiac magnetic resonance imaging (CMR) findings per study

\begin{tabular}{|c|c|c|c|c|c|c|c|c|c|}
\hline CMR finding & $\begin{array}{l}\text { Total } \\
\text { studies } \\
\text { (n) }\end{array}$ & $\begin{array}{l}\text { Gan, } \\
2007 \\
{[18]}\end{array}$ & $\begin{array}{l}\text { Van } \\
\text { Wolferen, } \\
2007[19]\end{array}$ & $\begin{array}{l}\text { Van de } \\
\text { Veerdonk, } \\
2011[20]\end{array}$ & $\begin{array}{l}\text { Freed, } \\
2012 \\
{[21]}\end{array}$ & $\begin{array}{l}\text { Yamada, } \\
2012 \\
{[22]}\end{array}$ & $\begin{array}{l}\text { Cho, } \\
2014 \\
{[23]}\end{array}$ & $\begin{array}{l}\text { Swift, } \\
2014 \\
{[24]}\end{array}$ & $\begin{array}{l}\text { Swift, } \\
2014 \\
{[25]}\end{array}$ \\
\hline RV ejection fraction & 6 & & $x^{a}$ & $x^{a}$ & $\mathrm{x}$ & $\mathrm{x}$ & $\mathrm{x}$ & $\mathrm{x}$ & \\
\hline RV end-diastolic volume & 5 & & $\mathrm{x}^{\mathrm{a}}$ & $x^{a}$ & $\mathrm{x}$ & $\mathrm{x}$ & & $\mathrm{x}$ & \\
\hline RV end-systolic volume & 4 & & & $x^{a}$ & $\mathrm{x}$ & $\mathrm{x}$ & & $\mathrm{x}$ & \\
\hline RV stroke volume & 3 & & & & $\mathrm{x}$ & $\mathrm{x}$ & & $\mathrm{x}$ & \\
\hline PA flow stroke volume index & 2 & & $x^{a}$ & & & & & $\mathrm{x}$ & \\
\hline PA flow cardiac index & 2 & & $x^{a}$ & & & & & $\mathrm{x}$ & \\
\hline RV mass & 3 & & $x^{a}$ & & $\mathrm{x}$ & $\mathrm{x}$ & & & \\
\hline LV ejection fraction & 5 & & $x^{a}$ & $x^{a}$ & $\mathrm{x}$ & $\mathrm{x}$ & & $\mathrm{x}$ & \\
\hline LV end-diastolic volume & 5 & & $x^{a}$ & $x^{a}$ & $\mathrm{x}$ & $\mathrm{x}$ & & $\mathrm{x}$ & \\
\hline LV end-systolic volume & 4 & & & $x^{a}$ & $\mathrm{x}$ & $\mathrm{x}$ & & $\mathrm{x}$ & \\
\hline LV stroke volume & 2 & & & $x^{a}$ & & & & $\mathrm{x}$ & \\
\hline LV mass & 3 & & $x^{a}$ & & $\mathrm{x}$ & $\mathrm{x}$ & & & \\
\hline RV wall thickness & 1 & & $x^{a}$ & & & & & & \\
\hline LV wall thickness & 1 & & $x^{a}$ & & & & & & \\
\hline Ventricular mass index (RV/LV mass) & 1 & & & & & & & $\mathrm{x}$ & \\
\hline RA volume & 1 & & & & $\mathrm{x}$ & & & & \\
\hline LA volume & 1 & & & & $\mathrm{x}$ & & & & \\
\hline PA relative area change & 1 & $\mathrm{x}$ & & & & & & & \\
\hline $\begin{array}{l}\text { RV insertion points late gadolinium } \\
\text { enhancement }\end{array}$ & 1 & & & & $\mathrm{x}$ & & & & \\
\hline $\begin{array}{l}\text { Full width at half maximum of the } \\
\text { bolus } \\
\text { passage }\end{array}$ & 1 & & & & & & & & $\mathrm{x}$ \\
\hline Pulmonary transit time & 1 & & & & & & & & $\mathrm{x}$ \\
\hline
\end{tabular}

$L V$ left ventricular, $P A$ pulmonary artery, $R V$ right ventricular

${ }^{a}$ Additional serial investigation of CMR finding (change between baseline and follow-up)

of studies used short-axis segmentation for the measurement of RV volumes [19-21, 23, 24]; one study used a transverse segmenting method [22] and none of the studies used axial slice segmentation. Slice thickness varied between 5 and $10 \mathrm{~mm}$ and temporal resolution between 20 and 25 frames/cycle or $35-45 \mathrm{~ms}$. Two studies did not report spatial or temporal resolution $[19,21]$. None of the studies explicitly described if the valvular planes were taken into account in the segmentation and how the tricuspid valve was delineated; however, most studies do mention that both ventricles were covered from base to apex. Most studies are not clear about the methodology used for the selection of the trabeculae. The technical details regarding the CMR acquisition and analysis are described in more detail in Supplemental File 2.

Although all studies reported HRs using Cox regression analysis, large differences were found regarding the type and number of predictors per event used in the multivariable analysis. Five studies performed some form of multivariable adjustment, of which only one study adjusted for age and sex. Only one study used ten or more events per predictor. Because of this large variety between studies and overall suboptimal methodological quality of multivariable adjustment, it was decided to present only univariable HRs in the forest plots.

\section{Prognostic value of baseline CMR findings}

Eight studies evaluated 21 different CMR findings, as shown in Table 3. Meta-analysis was performed for nine CMR findings that were evaluated by three or more studies. Forest plots and pooled HRs are presented in Fig. 2. The strongest predictor of mortality was RV ejection fraction: pooled HR 1.23 (95\% CI 1.07-1.41, p=0.003) per $5 \%$ decrease. In addition, $\mathrm{RV}$ end-diastolic volume index $(\mathrm{p}=0.049)$ and end-systolic volume index $(p=0.013)$ and left ventricular $(L V)$ enddiastolic volume index $(p=0.045)$ were of prognostic importance. Notably, measurements of RV and LV mass did not provide prognostic information in PAH $(\mathrm{p}=0.852$ and $\mathrm{p}=0.983$, respectively).

Although RV stroke volume index as measured by the summation of disks method did not have a significant 


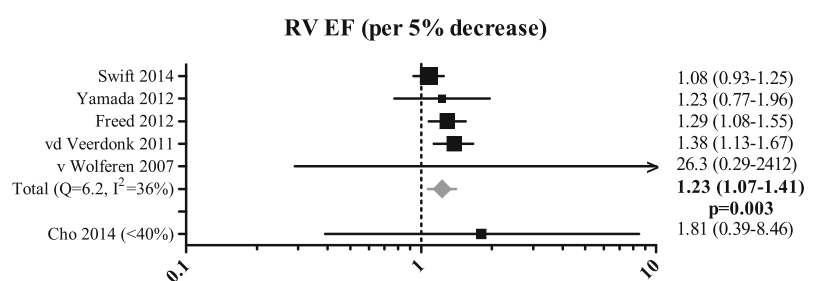

RV EDVI (per $5 \mathrm{~mL} / \mathrm{m}^{2}$ increase)

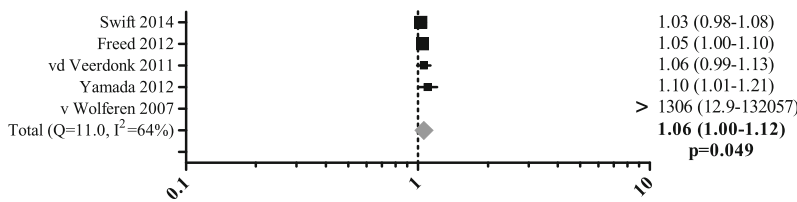

RV ESVI (per $5 \mathrm{~mL} / \mathrm{m}^{2}$ increase)

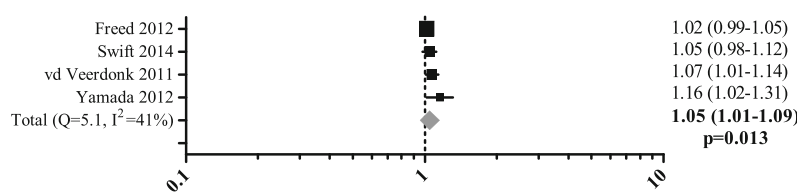

RV MI (per $5 \mathrm{gr} / \mathrm{m}^{2}$ increase)

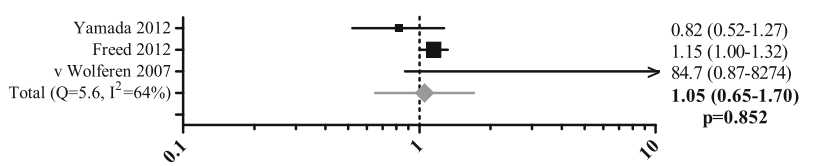

RV SVI (per $5 \mathrm{~mL} / \mathrm{m}^{2}$ decrease)

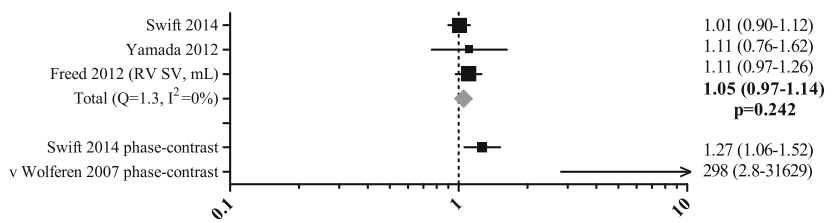

Fig. 2 Prognostic value of cardiac magnetic resonance imaging (CMR) findings evaluated by three or more studies. Values are presented as mean [95\% confidence interval]. EDVI end-diastolic volume index, $E F$

prognostic value (pooled HR 1.05, $\mathrm{p}=0.242$ ) $[21,22$, 24], the pulmonary artery (PA) flow stroke volume index as measured by CMR phase-contrast imaging predicted mortality in two studies [19, 24]. Less investigated CMR measurements such as LV stroke volume [20, 24], pulmonary artery relative area change [18], left atrial volume, late gadolinium enhancement at RV insertion points [21] and two novel dynamic contrast-enhanced CMR measurements (full width at half maximum of the bolus passage and pulmonary transit time) [25] seem promising but require further evaluation.

\section{Serial CMR evaluation}

Two studies included in this review additionally quantified the individual change in specific CMR findings during a followup period and directly associated these with patient outcomes.
LV EF (per 5\% decrease)

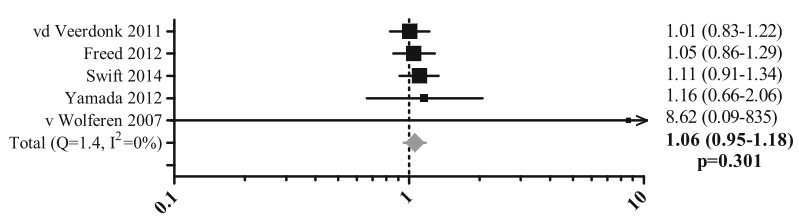

LV EDVI (per $5 \mathrm{~mL} / \mathrm{m}^{2}$ decrease)

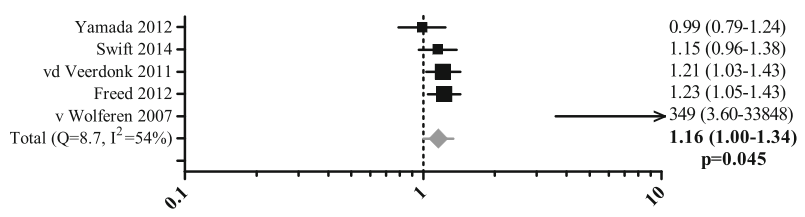

LV ESVI (per $5 \mathrm{~mL} / \mathrm{m}^{2}$ decrease)

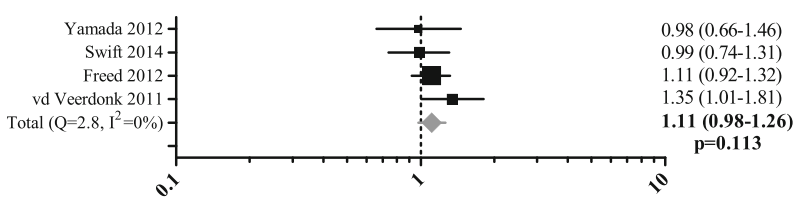

LV MI (per $5 \mathrm{gr} / \mathrm{m}^{2}$ increase)

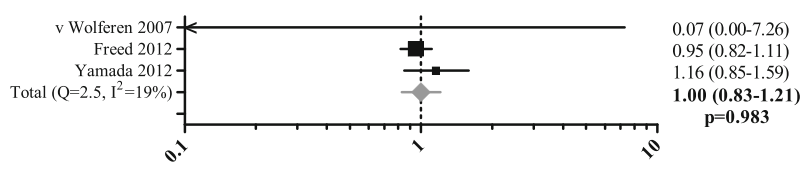

ejection fraction, ESVI end-systolic volume index, $L V$ left ventricular, $M I$ mass index, $R V$ right ventricular, $S V I$ stroke volume index

Van Wolferen et al. [19] showed that overall mortality was associated with a decrease in RV ejection fraction $(\mathrm{p}=0.015)$ and PA flow stroke volume index $(\mathrm{p}=0.006)$ at 1 -year followup. Van de Veerdonk's group [20] reported a 1-year change in RV ejection fraction ( $p=0.014), \mathrm{RV}$ end-diastolic and endsystolic volume index (both $\mathrm{p}<0.001$ ) as significant predictors of long-term outcome.

\section{Risk of bias assessment}

A combination of visual assessment of funnel plots and Egger's [17] test did not provide statistical evidence for publication bias for any of the CMR findings included in the metaanalysis. Statistical heterogeneity among studies was generally low, as presented by Cochran's $\mathrm{Q}$ and $\mathrm{I}^{2}$ statistics in Fig. 2 in the corresponding forest plots. We noted a discrepancy between the values that were reported in the study by van 
Wolferen et al. [19] compared to the other studies, as effect sizes and standard errors were extremely large in this study. We therefore conducted sensitivity analyses by excluding the results of this study. This did not result in significantly different HRs and did thus not change our conclusions.

\section{Discussion}

To our knowledge, this is the first systematic review and metaanalysis that substantiates the clinical yield of CMR findings in the prognostication of PAH patients. Among eight studies (539 patients) that investigated 21 different CMR findings, $\mathrm{RV}$ ejection fraction was found to be the strongest and most well established predictor of mortality in PAH. In addition, increased RV volumes and decreased LV end-diastolic volume at baseline were found to be associated with a higher mortality risk in PAH patients.

\section{Right heart failure}

The primary cause of death in PAH is right heart decompensation [8]. This is congruent with the observation that a decrease in RV function is such an important prognostic factor, as it indicates that the RV is no longer able to cope with the high pulmonary pressures, leading to increased RV diastolic pressures and RV dilatation. RV cardiac output as invasively assessed during right heart catheterization has similarly shown to predict mortality in PAH $[7,8]$. Studies that have investigated echocardiographic measurements of RV function, such as TAPSE or RV longitudinal strain, report similar findings $[26,27]$. RV ejection fraction assessed with equilibrium radionuclide angiography also showed to be of prognostic importance [28]. Nevertheless, CMR is currently considered as the reference standard for the assessment of RV volumes and function [29]. RV function as measured with CMR may therefore be the most accurate and thus most reliable predictor. However, it has to be acknowledged that direct comparisons between the different imaging modalities are not reported yet.

\section{Ventricular-ventricular interaction}

Whereas CMR-derived RV volumes are increased in high-risk PAH patients, LV end-diastolic volume is decreased. Similar results have been reported in several echocardiographic studies [30-32]. These findings emphasize even more the inextricable connection between the two ventricles. Although often considered as separate entities, the two ventricles share common myofibers, a noncompliant pericardium and of course the interventricular septum [33]. The pressure-overloaded RV will alter LV geometry by a leftward septal shift, resulting in a Dshaped LV, which is generally considered as one of the hallmarks of pulmonary hypertension. This so-called systolic D- sign is primarily an expression of RV pressure overload, in contrast to a diastolic D-sign which is considered a sign of a volume-overloaded RV [34]. Interestingly, previous studies have shown that the diastolic leftward septal shift is associated with mortality in $\mathrm{PAH}$, in contrast to the systolic leftward septal shift [35]. Accordingly, we found decreased LV enddiastolic volume to be of prognostic value, in contrast to LV end-systolic volume.

The overall observation that systolic LV geometry is not associated with mortality may be explained by the fact that RV systolic pressures are generally high in a stable PAH state. However, once the RV starts to fail, LV preload will decrease due to lower transpulmonary flow, and RV diastolic pressures will rise. The accompanied altered diastolic LV geometry can therefore be seen as a direct expression of RV failure [35]. Subsequently, this altered shape could impair LV filling and function, further leading to increased pulmonary pressures, which may create a vicious circle tilting a stable PAH state towards clinical deterioration and, ultimately, death.

\section{Serial measurements}

Apart from baseline findings evaluated at the time of diagnosis, changes in several haemodynamic, functional, biochemical and imaging variables could carry incremental prognostic information, as they conceivably better reflect an individual's course of disease [19, 20, 36, 37]. Thus far, only two studies have directly related subject-specific changes in CMR measurements to clinical outcome using Cox regression [19, 20]. Both studies report the prognostic importance of a decrease in $\mathrm{RV}$ ejection fraction over time. In addition, van de Veerdonk et al. [38] recently published the results of repeated CMR measurements during a follow-up period of 10 years in patients with initially stable idiopathic PAH for 5 years. Although no HRs were presented, this study showed that late disease progression is also accompanied by increased RV volumes and decreased RV ejection fraction.

\section{RV mass}

Based on three studies, our results showed that RV mass does not provide prognostic information in PAH [19, 21, 22]. This is probably because concentric RV hypertrophy is an adaptive response to increased RV pressures that serves to maintain wall stress as low as possible, as is also seen in the $\operatorname{LV}[39,40]$.

$\mathrm{RV}$ mass-to-volume ratio allows the distinction between RV concentric and eccentric hypertrophy, which might better reflect the RV's adaptive or maladaptive response. A recently published cross-sectional study in patients with idiopathic PAH showed that eccentric hypertrophy, reflected by a lower RV mass-to-volume ratio, was clearly related to worse RV systolic function, right ventricular to arterial coupling and 
clinical impairment [40]. Moreover, in patients with tetralogy of Fallot, a lower RV mass-to-volume ratio was predictive of death [41]. Therefore, it may be more prudent to focus on RV mass-to-volume ratios rather than on RV mass alone.

\section{Study limitations}

Although we calculated pooled effect estimates in our review, the results of the random effect models should be interpreted with caution, as we included only univariable HRs in the meta-analysis, due to the large variability between studies in which multivariable adjustment was performed (regarding the type and number of predictors per event used). Second, although formal tests for publication bias yielded mainly nonsignificant results, the relative lack of power of Egger's test in detecting publication bias for imaging findings investigated in less than ten studies should be recognized. Theoretically, publication bias can cause under-reporting of non-significant HRs, leading to a relative overestimation of the pooled HRs. Finally, although differences within studies regarding the CMR acquisition and analysis probably reflect the normal variance in day-to-day practice, they may lead to less precise measurements (independent of the outcome). While the vast majority of the studies used short-axis segmentation, it has been suggested that axial segmentation results in higher reproducibility, as it may decrease the difficulty of valve delineation in the basal slices [42, 43]. Incomplete segmentation of the tricuspid plane, inaccurate delineation of the basal slice and the methodology used to deal with the trabeculae can substantially impact the measurements of RV volumes [44, 45]. The subsequent 'random noise' would dilute the effect and thus could cause a relative underestimation of the actual hazard ratios.

\section{Clinical implications}

This review substantiates the clinical yield of specific CMR findings in the identification of patients with PAH at higher risk of clinical deterioration. This is important, as timely intensification of $\mathrm{PAH}$-specific therapy could prevent further clinical worsening and death. In addition, CMR-derived RV ejection fraction could be of additional value for the longitudinal assessment of PAH. More research is needed to investigate the prognostic value of other serial CMR measurements, and baseline measurements such as RV mass-to-volume ratio, LV stroke volume, pulmonary artery relative area change and left atrial volume. In addition, it would be worthwhile to directly compare the prognostic value of RV function measured using CMR with RV function measured using other non-invasive imaging modalities, such as echocardiography.

\section{Conclusion}

CMR is useful and accurate in the prognostication of PAH patients. RV ejection fraction at baseline and during followup is the strongest and most well investigated predictor of mortality in patients with PAH. In addition, increased RV volumes and decreased LV end-diastolic volume are of prognostic importance.

Acknowledgments The scientific guarantor of this publication is Dr. G.Tj. Sieswerda. The authors of this manuscript declare no relationships with any companies whose products or services may be related to the subject matter of the article. The authors state that this work has not received any funding. Prof. Dr. E. Boersma has significant statistical expertise. Institutional Review Board approval was not required because as this is a systematic review, no human subjects are directly involved. This is a systematic review and meta-analysis of both prospective and retrospective prognostic studies performed at one institution. Systematic review registration number: CRD42014009231.

Open Access This article is distributed under the terms of the Creative Commons Attribution-NonCommercial 4.0 International License (http:// creativecommons.org/licenses/by-nc/4.0/), which permits any noncommercial use, distribution, and reproduction in any medium, provided you give appropriate credit to the original author(s) and the source, provide a link to the Creative Commons license, and indicate if changes were made.

\section{References}

1. Galie N, Hoeper MM, Humbert M et al (2009) Guidelines for the diagnosis and treatment of pulmonary hypertension: the Task Force for the Diagnosis and Treatment of Pulmonary Hypertension of the European Society of Cardiology (ESC) and the European Respiratory Society (ERS), endorsed by the International Society of Heart and Lung Transplantation (ISHLT). Eur Heart J 30:24932537

2. Simonneau G, Gatzoulis MA, Adatia I et al (2013) Updated clinical classification of pulmonary hypertension. J Am Coll Cardiol 62: D34-D41

3. Humbert M, Sitbon O, Chaouat A et al (2010) Survival in patients with idiopathic, familial, and anorexigen-associated pulmonary arterial hypertension in the modern management era. Circulation 122: 156-163

4. Humbert M, Lau EM, Montani D, Jais X, Sitbon O, Simonneau G (2014) Advances in therapeutic interventions for patients with pulmonary arterial hypertension. Circulation 130:2189-2208

5. McLaughlin VV (2013) Managing pulmonary arterial hypertension and optimizing treatment options: prognosis of pulmonary arterial hypertension. Am J Cardiol 111:11C-15C

6. McLaughlin VV, Presberg KW, Doyle RL et al (2004) Prognosis of pulmonary arterial hypertension. Chest 126:78S-92S

7. Benza RL, Miller DP, Gomberg-Maitland M et al (2010) Predicting survival in pulmonary arterial hypertension: insights from the Registry to Evaluate Early and Long-Term Pulmonary Arterial Hypertension Disease Management (REVEAL). Circulation 122: 164-172 
8. D'Alonzo GE, Barst RJ, Ayres SM et al (1991) Survival in patients with primary pulmonary hypertension. Results from a national prospective registry. Ann Intern Med 115:343-349

9. Fijalkowska A, Kurzyna M, Torbicki A et al (2006) Serum Nterminal brain natriuretic peptide as a prognostic parameter in patients with pulmonary hypertension. Chest 129:1313-1321

10. Wensel R, Opitz CF, Anker SD et al (2002) Assessment of survival in patients with primary pulmonary hypertension: importance of cardiopulmonary exercise testing. Circulation 106:319-324

11. Moher D, Liberati A, Tetzlaff J, Altman DG, Group P (2009) Preferred reporting items for systematic reviews and meta-analyses: the PRISMA statement. Ann Intern Med 151:264-269, W264

12. Gomberg-Maitland M, Bull TM, Saggar R et al (2013) New trial designs and potential therapies for pulmonary arterial hypertension. J Am Coll Cardiol 62:D82-D91

13. Geersing GJ, Bouwmeester W, Zuithoff P, Spijker R, Leeflang M, Moons K (2012) Search filters for finding prognostic and diagnostic prediction studies in Medline to enhance systematic reviews. PLoS ONE 7:e32844

14. Ingui BJ, Rogers MAM (2001) Searching for clinical prediction rules in MEDLINE. J Am Med Inform Assoc 8:391-397

15. Hayden JA, Cote P, Bombardier C (2006) Evaluation of the quality of prognosis studies in systematic reviews. Ann Intern Med 2006: $427-437$

16. Higgins JPT, Thompson SG, Deeks JJ, Altman DG (2003) Measuring inconsistency in meta-analyses. BMJ 327:557-560

17. Egger M, Davey Smith G, Schneider M, Minder C (1997) Bias in meta-analysis detected by a simple, graphical test. BMJ 315:629-634

18. Gan CTJ, Lankhaar JW, Westerhof N et al (2007) Noninvasively assessed pulmonary artery stiffness predicts mortality in pulmonary arterial hypertension. Chest 132:1906-1912

19. van Wolferen SA, Marcus JT, Boonstra A et al (2007) Prognostic value of right ventricular mass, volume, and function in idiopathic pulmonary arterial hypertension. Eur Heart J 28:1250-1257

20. Van de Veerdonk MC, Kind T, Marcus JT et al (2011) Progressive right ventricular dysfunction in patients with pulmonary arterial hypertension responding to therapy. J Am Coll Cardiol 58:25112519

21. Freed BH, Gomberg-Maitland M, Chandra S et al (2012) Late gadolinium enhancement cardiovascular magnetic resonance predicts clinical worsening in patients with pulmonary hypertension. $\mathrm{J}$ Cardiovasc Magn Reson 14:11

22. Yamada Y, Okuda S, Kataoka M et al (2012) Prognostic value of cardiac magnetic resonance imaging for idiopathic pulmonary arterial hypertension before initiating intravenous prostacyclin therapy. Circ J 76:1737-1743

23. Cho IJ, Oh J, Chang HJ et al (2014) Tricuspid regurgitation duration correlates with cardiovascular magnetic resonance-derived right ventricular ejection fraction and predict prognosis in patients with pulmonary arterial hypertension. Eur Heart J Cardiovasc Imaging $15: 18-23$

24. Swift AJ, Rajaram S, Campbell MJ et al (2014) Prognostic value of cardiovascular magnetic resonance imaging measurements corrected for age and sex in idiopathic pulmonary arterial hypertension. Circ Cardiovase Imaging 7:100-106

25. Swift AJ, Telfer A, Rajaram S et al (2014) Dynamic contrastenhanced magnetic resonance imaging in patients with pulmonary arterial hypertension. Pulm Circ 4:61-70

26. Forfia PR, Fisher MR, Mathai SC et al (2006) Tricuspid annular displacement predicts survival in pulmonary hypertension. Am J Respir Crit Care Med 174:1034-1041

27. Sachdev A, Villarraga HR, Frantz RP et al (2011) Right ventricular strain for prediction of survival in patients with pulmonary arterial hypertension. Chest 139:1299-1309
28. Courand PY, Pina Jomir G, Khouatra C et al (2015) Prognostic value of right ventricular ejection fraction in pulmonary arterial hypertension. Eur Respir J 45:139-149

29. Baumgartner H, Bonhoeffer P, De Groot NM et al (2010) ESC guidelines for the management of grown-up congenital heart disease (new version 2010). Eur Heart J 31:2915-2957

30. Ghio S, Klersy C, Magrini G et al (2010) Prognostic relevance of the echocardiographic assessment of right ventricular function in patients with idiopathic pulmonary arterial hypertension. Int $\mathrm{J}$ Cardiol 140:272-278

31. Giusca S, Jurcut R, Coman IM et al (2013) Right ventricular function predicts clinical response to specific vasodilator therapy in patients with pulmonary hypertension. Echocardiography 30:17-26

32. Tonelli AR, Conci D, Tamarappoo BK, Newman J, Dweik RA (2014) Prognostic value of echocardiographic changes in patients with pulmonary arterial hypertension receiving parenteral prostacyclin therapy. J Am Soc Echocardiogr 27:733-741, e2

33. Haddad F, Doyle R, Murphy DJ, Hunt SA (2008) Right ventricular function in cardiovascular disease, part II: pathophysiology, clinical importance, and management of right ventricular failure. Circulation 117:1717-1731

34. Ryan T, Petrovic O, Dillon JC, Feigenbaum H, Conley MJ, Armstrong WF (1985) An echocardiographic index for separation of right ventricular volume and pressure overload. J Am Coll Cardiol 5:918-927

35. Raymond RJ, Hinderliter AL, Willis PW et al (2002) Echocardiographic predictors of adverse outcomes in primary pulmonary hypertension. J Am Coll Cardiol 39:1214-1219

36. Provencher S, Sitbon O, Humbert M, Cabrol S, Jais X, Simonneau $\mathrm{G}$ (2006) Long-term outcome with first-line bosentan therapy in idiopathic pulmonary arterial hypertension. Eur Heart J 27:589-595

37. Nickel N, Golpon H, Greer M et al (2012) The prognostic impact of follow-up assessments in patients with idiopathic pulmonary arterial hypertension. Eur Respir J 39:589-596

38. van de Veerdonk MC, Marcus JT, Westerhof N et al (2015) Signs of right ventricular deterioration in clinically stable patients with pulmonary arterial hypertension. Chest 147:1063-1071

39. Drazner MH (2011) The progression of hypertensive heart disease. Circulation 123:327-334

40. Badagliacca R, Poscia R, Pezzuto B et al (2015) Right ventricular remodeling in idiopathic pulmonary arterial hypertension: adaptive versus maladaptive morphology. J Heart Lung Transplant 34:395403

41. Valente AM, Gauvreau K, Assenza GE et al (2014) Contemporary predictors of death and sustained ventricular tachycardia in patients with repaired tetralogy of Fallot enrolled in the INDICATOR cohort. Heart 100:247-253

42. Schelhorn J, Neudorf U, Schemuth H, Nensa F, Nassenstein K, Schlosser TW (2015) Volumetric measurements in patients with corrected tetralogy of Fallot: comparison of short-axis versus axial cardiac MRI and echocardiography. Acta Radiol 56:1315-1322

43. Clarke CJ, Gurka MJ, Norton PT, Kramer CM, Hoyer AW (2012) Assessment of the accuracy and reproducibility of RV volume measurements by CMR in congenital heart disease. JACC Cardiovasc Imaging 5:28-37

44. Caudron J, Fares J, Lefebvre V, Vivier PH, Petitjean C, Dacher JN (2012) Cardiac MRI assessment of right ventricular function in acquired heart disease: factors of variability. Acad Radiol 19:9911002

45. Driessen MM, Baggen VJ, Freling HG et al (2014) Pressure overloaded right ventricles: a multicenter study on the importance of trabeculae in RV function measured by CMR. Int J Cardiovasc Imaging 30:599-608 